\title{
Sindrome da queda de grande altura em gatos: 43 casos atendidos no município do Rio de Janeiro
}

\author{
Feline high-rise syndrome: 43 cases evaluated in the \\ Municipality of Rio de Janeiro
}

\author{
Margarete Weinschutz Gheren ${ }^{1 *}$, Amanda Chaves de Jesus ${ }^{1}$, Raquel Sampaio Alves ${ }^{1} \&$ \\ Heloisa Justen Moreira de Souza² \\ 'Médicas veterinárias, MSc. Programa de Pós-graduação em Medicina Veterinária, Universidade Federal Rural do Rio de Janeiro \\ - UFRRJ, Seropédica, RJ, Brasil \\ 2Médica veterinária, Dr. Departamento de Medicina e Cirurgia Veterinária, Instituto de Veterinária, Universidade Federal Rural \\ do Rio de Janeiro - UFRRJ, Seropédica, RJ, Brasil
}

\section{Resumo}

Este trabalho foi realizado em clínica veterinária particular, exclusiva no atendimento de gatos em Botafogo, no Rio de Janeiro. Foram atendidos 43 gatos que sofreram queda de grande altura no período de janeiro de 2010 a dezembro de 2012 , tendo as quedas ocorrido entre $2^{\circ}$ e $12^{\circ}$ andares. A idade média dos animais atendidos foi de 2 anos e 9 meses e a altura média das quedas foi de 4,6 metros. Verificou-se que $72,42 \%$ dos gatos atendidos eram sem raça definida e 60,47\% eram machos. Não foi verificada uma prevalência na ocorrência das quedas de acordo com períodos do dia. Embora não seja estatisticamente significativo, houve uma maior ocorrência de quedas nos fins de semana quando comparado aos dias da semana. As quedas ocorreram em proporções semelhantes em todas as estações do ano não tendo sido notada uma sazonalidade na ocorrência de queda, como ocorre em países de clima temperado. Em 86,05\% dos casos, as quedas ocorreram em residências que não possuíam nenhuma janela telada ou em que somente algumas janelas eram teladas. As quedas também ocorreram em residências que tinham todas as janelas teladas, representando 13,95\% do número de quedas. Em 35/43 casos, as quedas ocorreram de altura equivalente a até o 60 andar e somente $8 / 43$ casos ocorreram de altura equivalente ou superior ao 70 andar. Foi observado fratura de membro torácico em 41,86\% dos casos; fratura de membro pélvico em 30,23\%, luxação de membro torácico e de membro pélvico em 4,65\% dos casos cada; fratura e luxação de coluna 2,33\% e 9,30\% respectivamente; fratura de pelve 4,65\%; fratura de sínfise 6,98\%; fratura de mandíbula 6,98\%; luxação de articulação temporomandibular 9,30\%; fístula oronasal 18,60\%; lesão na língua 11,62\%; escoriações na face $44,18 \%$. Ainda foram observados epistaxe em 25,58\%; choque 6,97\%, hipotermia 11,62\%; hipotensão 4,65\%; dispneia 18,60\%; trauma torácico 41,86\%, sendo 38,88\% pneumotórax e 61,11\% contusão pulmonar. Ainda foram verificadas lesões em órgãos do sistema esplâncnico em 6,98\% dos casos. Dos 43 gatos que foram atendidos, 51,16\% receberam tratamento emergencial, 44,19\% não emergencial e 6 vieram a óbito, sendo que 2/6 (4,65\%) já chegaram mortos. Concluímos que os animais jovens estão mais sujeitos ao risco de queda e não há um período do dia em que as quedas ocorram com maior frequência. Por estarmos em um país de clima tropical, onde as temperaturas não variam tanto ao longo do ano, não foi observada uma sazonalidade na ocorrência de quedas. Embora as fraturas e lesões na face ocorram com frequência nas quedas de grande altura, devemos sempre estar atentos para identificar lesões pulmonares, pois estas quando não adequadamente tratadas, podem levar os gatos a óbito, como também, as lesões em órgãos do sistema esplâncnico.

Palavras-chave: queda de grande altura, gatos, Rio de Janeiro.

\footnotetext{
Abstract

This study was done in a private veterinary clinic, specialized in cats in Botafogo, Rio de Janeiro. We evaluated 43 cats diagnosed with high-rise syndrome from January 2010 to December 2012. The cats fell from the $2^{\text {nd }}$ to the $12^{\text {th }}$ floor. The average age of the cats was 2 years and 9 months and the average height of the fall was 4.6 meters. From the diagnosed cats $72.42 \%$ were mixed-breed and $60.47 \%$ were male. The falls happened at any time of the day and, although it was not statistically significant, they happened more frequently during the weekends when compared to the weekdays. The falls occurred in similar proportion through out the year not being noticed a seasonal incidence as it happens in temperate climates. In $86.05 \%$ of the cases, the falls happened from homes where none of the windows
}

Como citar: Gheren, M. W., Jesus, A. C., Alves, R. S., \& Souza, H. J. M. (2017). Síndrome da queda de grande altura em gatos: 43 casos atendidos no município do Rio de Janeiro. Brazilian Journal of Veterinary Medicine, 39(3) 182-189. doi: 10.29374/2527-2179.bjvm026016

Fonte de financiamento: Nenhuma.

Conflito de interesses: Os autores declaram não haver conflito de interesses que precisam ser informados.

Recebido: Agosto 26, 2016.

Aceito: Janeiro 07, 2017.

O estudo foi realizado em clínica veterinária particular, especializada no atendimento de felinos, localizada no bairro de Botafogo, na cidade do Rio de Janeiro, RJ, Brasil.

\section{${ }^{*}$ Correspondência}

Margarete Weinschutz Gheren Programa de Pós-graduação em Medicina Veterinária, Universidade Federal Rural do Rio de Janeiro - UFRRJ

Rodovia BR 465, Km 07, s/n, Zona Rural CEP 23890-000 - Seropédica (RJ), Brasil E-mail:margagheren@yahoo.com.br
Copyright Gheren et al. Este é um artigo publicado em acesso aberto (Open Access) sob a licença Creative Commons Attribution Non-Commercial, que permite uso, distribuição e reprodução em qualquer meio, sem restrições desde que sem fins comerciais e que o trabalho original seja corretamente citado. 
had screens. And 13.95\% of the falls happened from homes where all the windows had screens. The falls occurred from heights equivalent to up to the $6^{\text {th }}$ floor in $35 / 43$ of the cases and only $8 / 43$ falls occurred from height equivalent to the $7^{\text {th }}$ floor on. $41.86 \%$ of the cats had forelimb fractures; $30.23 \%$ had hindlimb fracture; $4.65 \%$ had forelimb and hindlimb luxation each; $2.33 \%$ had spine fracture and 9.30\% had spine luxation; $4.65 \%$ had pelvic fracture; $6.98 \%$ had fracture of the symphysis; $6.98 \%$ had fracture of the mandible; $9.30 \%$ had luxation of the temporomandibular joint; $18.60 \%$ had hard palate fractures; $11.62 \%$ had tongue injury and $44.18 \%$ had facial injury. It was also observed epistaxis in $22.58 \%$ of the cases; shock in 6.97\%; hypothermia in 11.62\%; hypotension in 4.65\%; dyspnea in 18.6\%; thoracic trauma in $41.86 \%$, being $38.88 \%$ pneumothorax and $61.11 \%$ pulmonary contusion. It was also diagnosed visceral injury in $6.98 \%$ of the cases. From the 43 diagnosed cats, 51.16 had emergency treatment and $44.19 \%$ had non-emergency treatment. Six cats died. From these six, two (4.65\% arrived dead. We can conclude that young cats are more liable to the risk of falling and the falls can occur at any time. As we live in a tropical climate where the temperatures don" $t$ vary much through out the year, a seasonal incidence is not related to the falls. In high-rise syndrome fractures and facial injure are frequently noticed, however, we must be aware to identify pulmonary trauma because when they are not properly treated, they can be the cause of death as well as visceral injure.

Keywords: high-rise syndrome, cats, Rio de Janeiro.

\section{Introdução}

O termo Síndrome da Queda de Grande Altura (High-rise Syndrome) é utilizado para descrever injúrias sofridas por gatos que caem de janelas ou sacadas de prédios e casas, sendo a altura mínima da queda equivalente a dois andares. Deve-se levar em consideração que cada andar corresponde a 3,7 metros (Robinson, 1976; Whitney \& Mehlhaff, 1987; Faria, 2003; Boundrieau, 2004; Vnuk et al., 2004; Crystal, 2009).

Este termo apareceu na literatura veterinária em 1976, com a publicação do primeiro relato de caso em um gato. Entretanto, somente em 1987 surgiu o primeiro estudo retrospectivo, incluindo 132 gatos. Foi observado que um grupo de lesões se repetia com freqüência nos pacientes, compondo assim, o que pode ser chamado de síndrome (Whitney \& Mehlhaff, 1987; Faria, 2003).

A síndrome da queda de grande altura foi caracterizada pela tríade de injúrias que inclui epistaxi, fratura de palato duro e pneumotórax. Esta tríade é frequentemente acompanhada de outras injúrias que incluem trauma torácico, sendo contusão pulmonar e pneumotórax os mais frequentes, trauma facial, fratura de canino, fratura de sínfise mandibular, luxação de mandíbula, fratura de fêmur (normalmente distal), fratura de radio e ulna, fratura do colo do fêmur em gatos jovens, fratura distal de tíbia, fraturas de extremidades, hipotermia e choque e luxações traumáticas (Robinson, 1976; Whitney \& Mehlhaff, 1987; Pratschke \& Kirby, 2002; Faria, 2003; Boundrieau, 2004).

A maioria dos gatos envolvidos em quedas de grande altura é relativamente jovem, normalmente tem menos de três anos, o que explica sua inexperiência em relação ao ambiente e sua natureza curiosa colocando-os em situação de risco (Whitney \& Mehlhaff, 1987; Faria, 2003; Boundrieau, 2004; Vnuk et al., 2004).

A síndrome da queda de grande altura tem sido avaliada em diversos países de clima temperado, onde as estações do ano são bem definidas e, portanto, uma sazonalidade é observada na ocorrência das mesmas. Também é citado em alguns trabalhos, que as quedas ocorrem mais a noite por ser o período em que os gatos tem maior atividade e as pessoas estão em casa.

Entretanto, nenhum estudo foi feito em países de clima tropical. Portanto, não sabemos se uma sazonalidade também pode ser observada no Rio de Janeiro, onde as temperaturas são altas na maior parte do ano e onde as estações não são bem definidas. Não temos dados sobre como esta síndrome ocorre no Rio de Janeiro, tão pouco sobre o perfil dos gatos envolvidos e de seus proprietários.

Também é importante pontuarmos os tipos de lesões que ocorrem, verificando não somente as fraturas e lesões em face, mas principalmente as lesões torácicas e em sistema esplâncnico. É de fundamental importância que o animal que sofre queda de grande altura seja devidamente tratado, priorizando-se sempre o sistema respiratório e cardiovascular. 
Portanto, devemos estar atentos para os diversos tipos de lesões envolvidas na síndrome da queda de grande altura e trata-las de forma adequada e em ordem de gravidade e importância, sempre com o objetivo de salvar a vida do animal envolvido.

O objetivo deste trabalho, é então, identificar fatores que possam predispor a ocorrência de quedas, verificar a ocorrência de sazonalidade em clima tropical e alertar para os tipos de lesões que podem ocorrer além das fraturas.

\section{Material e métodos}

O projeto foi realizado em clínica veterinária particular, exclusiva no atendimento de gatos, localizada no bairro de Botafogo na cidade do Rio de Janeiro, onde foram obtidos os dados dos gatos que participaram do estudo casuístico, no período de janeiro de 2010 a dezembro de 2012.

Foram admitidos no estudo gatos domésticos (Felis catus), machos e fêmeas, esterilizados ou não, sem determinação de raça nem de idade atendidos na rotina clínica que sofreram queda de altura mínima equivalente a dois andares.

O histórico e anamnese dos animais foram coletados identificando o sexo, raça, idade, estado reprodutivo, tipo de moradia, época do ano e altura da queda, após estabilização do quadro clínico do paciente.

Dados referentes à altura da queda e piora ou não do estado geral do gato desde que foi encontrado até chegar à clínica, eram obtidos pelo médico veterinário durante a anamnese.

O exame físico foi feito seguindo um padrão de prioridades onde foi verificado primeiramente sistemas respiratório e cardiovascular. As alterações respiratórias foram sempre priorizadas e a estabilização do animal era feita antes de dar sequência ao exame clínico. Em seguida, foram avaliadas lesões abdominais, tegumentares e ósseas, dentre outras, de acordo com a gravidade do caso. Os exames complementares foram solicitados, mediante a necessidade da investigação clínica e após a estabilização do paciente.

Os proprietários responderam um questionário (Figura 1) onde forneceram dados do animal como idade, se castrados ou não, se estavam no cio; e detalhes específicos relativos ao acidente como hora e andar da queda, superfície de aterrissagem, se todas as janelas eram teladas, ocorrência de alteração na rotina da casa, se havia episódio de queda anterior e quanto à presença de pessoas em casa no momento da queda.

Este questionário, também, foi respondido pelo veterinário que prestou atendimento ao animal, fornecendo informações sobre o tipo de tratamento prestado (emergencial ou não e importantes observações), estado geral do animal, se apresentava alterações clínicas como hipotermia, choque, hipotensão, dispneia, cianose, hemorragia, tipo e local de fraturas e /ou luxações, presença de trauma torácico como hemotórax, contusão pulmonar e/ou pneumotórax, lesões encontradas em sistema esplâncnico e ocorrência de lesões em boca e face. Igualmente, foram anotados no questionário quais exames complementares foram realizados e se o gato veio ou não a óbito.

De acordo com o caso e a necessidade, após estabilização do paciente, eram realizados estudo radiológico do tórax, abdome, membros, cabeça e/ou coluna; estudo hematológico e bioquímica sérica, e estudo ultrassonográfico abdominal.

Para avaliar a ocorrência de queda em relação ao período do dia, as quedas foram divididas em dois grupos: as que ocorreram durante o dia e as que ocorreram durante a noite. As quedas que ocorreram entre às 6:00 horas e às 18:00 horas, foram consideradas como tendo ocorrido durante o dia. E as quedas que ocorreram entre 19:00 horas e 5:00 horas, foram consideradas como tendo ocorrido a noite.

Quanto às lesões, os animais foram divididos em 2 grupos: animais que sofreram queda de altura equivalente a até $06^{\circ}$ andar e animais que sofreram queda de altura equivalente ao 70 andar em diante.

Os resultados foram apresentados com o apoio da Estatística Descritiva.

A Estatística Inferencial também foi aplicada, por meio do teste $\chi^{2}$, que é um teste não paramétrico, utilizando o nível de significância de 5\% de probabilidade para rejeitar as diversas hipóteses formuladas $(\alpha=0,05)$. 


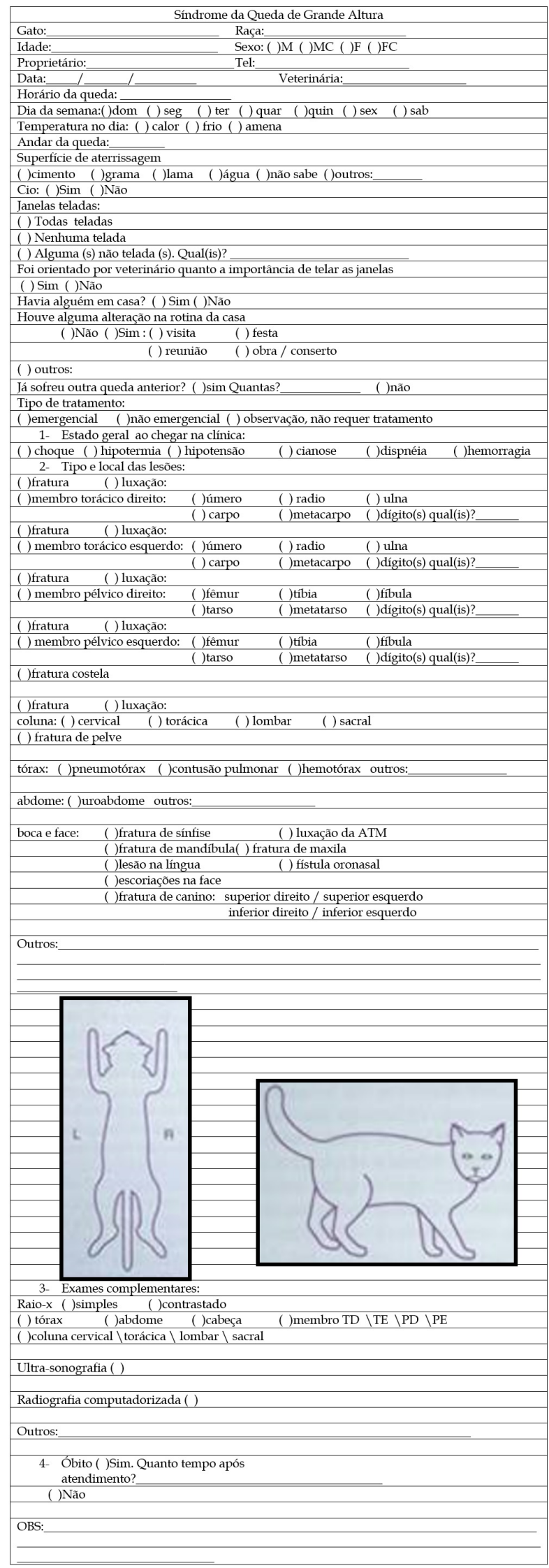

Figura 1. Questionário destinado ao proprietário e ao veterinário. 


\section{Resultados}

A pesquisa foi efetuada com uma amostra de 43 felinos com idade entre 4 meses e 12 anos, com média de idade de 2 anos e 9 meses, que foram atendidos em clínica exclusiva no atendimento de gatos, na Cidade do Rio de Janeiro, e que sofreram queda de altura média de 4,65 m.

A maior percentagem $(32 / 43=74,42 \%)$ de gatos que sofreram queda são SRD. No entanto, esta raça é a que mais ocorre na cidade do Rio de Janeiro.

Dos animais que sofreram queda, 26/43 = 60,47\% são machos enquanto que 39,53\% são fêmeas.

Com relação aos percentuais de felinos castrados $(20 / 43=46,51 \%)$ e não castrados $(23 / 43=53,49 \%)$, o teste $\chi^{2}$ igual a 0,21 não mostrou evidências para dizer que a castração aumenta ou diminui o percentual de queda entre os felinos.

O número de quedas dos felinos durante o dia foi de $21 / 43(48,84 \%)$ e durante a noite foi de $22 / 43$ (51,16\%), sendo que o teste $\chi^{2}$ igual a 0,02 não mostrou evidências para dizer que os percentuais de queda são influenciados pelo período do dia.

Em relação aos dias da semana, 28/43 ocorreram nos dias de semana, tendo uma média de 5,6 e 15/43 ocorreram nos fins de semana, tendo uma média de 7,5. Embora a média de queda diária seja maior nos finais de semana (sábado/domingo), este valor não é estatisticamente superior à média dos dias úteis.

As quedas aconteceram em proporções semelhantes durante todas as estações do ano, não sendo notada influência sazonal.

Neste trabalho, verificamos que ocorreram quedas mesmo em residências que tinham todas as janelas teladas

As quedas ocorreram de alturas equivalentes a até o 60 andar em 35/43 (81,40\%) dos casos. Apenas 8/43 (18,60\%) dos gatos sofreram queda de altura equivalente ou superior ao 70 andar.

Foram observadas fraturas de membro torácico em 18/43 (41,86\%) dos casos, sendo que $6 / 18$ (33,33\%) foram observadas em gatos que sofreram queda de altura acima do 60 andar. Em 1/43 caso foi observado fratura de escápula.

As fraturas de membro pélvico foram observadas em 13/43 (30,23\%) dos casos, sendo 4/13 (30,77\%) de andar superior ao 60 andar. As luxações de membro torácico foram observadas em 2/43 (4,65\%) dos casos e as de membro pélvico também em 2/43 (4,65\%) dos casos.

Essas lesões foram observadas somente em gatos que sofreram queda de altura equivalente a até o 60 andar. Este tipo de injúria está relacionado a quedas de altura equivalente a até seis andares, tendo sido a altura média das quedas de 4,6 andares.

Em apenas 1/43 (2,33\%) caso foi observado fratura de coluna e a queda se deu do 100 andar. As luxações de coluna foram observadas em 4/43 (9,30\%) casos e estas se deram de quedas equivalentes a até o $6^{\circ}$ andar. Ocorreram $2 / 43$ (4,65\%) casos de fratura de pelve sendo ambos resultantes de quedas do $10^{\circ}$ e $11^{\circ}$ andares respectivamente.

As fraturas de sínfise foram observadas em 3/43 (6,98\%) casos, sendo 1/43 decorrente de queda acima do 60 andar. As fraturas de mandíbula ocorreram em 3/43 (6,98\%) casos sendo todas decorrentes de quedas até o 60 andar.

Luxação de articulação têmporo-mandibular (ATM) ocorreu em 4/43 (9,30\%), sendo1/43 decorrente de queda acima do 60 andar e as outras $3 / 43$, de queda até o 60 andar. Apenas $2 / 43$ gatos apresentaram fratura de canino tendo estes sofrido queda de altura equivalente a até o $6^{\circ}$ andar.

Fístula oronasal foi observada em $8 / 43(18,60 \%)$, sendo que apenas $1 / 8$ foi decorrente de altura superior ao 60 andar. Lesão na língua foi verificada em $5 / 43$, sendo $1 / 5$ decorrente de queda acima do $6^{\circ}$ andar. Escoriações na face ocorreu em 19/43, sendo 1/19 decorrente de queda acima do 60 andar.

Ainda foram observados epistaxe em $11 / 43$ (25,58\%) casos, sendo 3/11 decorrente de queda acima do 60 andar; choque em $3 / 43$, sendo $2 / 3$ decorrente de queda acima do 60 andar; hipotermia em 5/43, sendo 2/5, decorrente de queda acima do 60 andar; hipotensão em $2 / 43$ casos, sendo $1 / 2$ do 50 e 1/2 do $10^{\circ}$ andar. Dispnéia ocorreu em 8/43 casos sendo 3/8 decorrentes de queda acima do 60 andar. 
Os traumas torácicos ocorreram em 18/43 (41,86\%) casos, sendo 7/43 pneumotórax e 11/43 contusão pulmonar. Dos casos de pneumotórax, 4/7 foram decorrentes de quedas acima do 60 andar; e 4/11 casos de contusão pulmonar também foram decorrentes de quedas acima do 60 andar.

Lesões em órgãos do sistema esplâncnico ocorreram em 3/43 (6,98\%) casos. Este tipo de lesão não foi relatado anteriormente. Insuficiência renal aguda (IRA) ocorreu em 2/43 casos sendo um caso de altura até o 60 andar e um acima do 60; e pancreatite em 1/43 casos decorrente de queda acima do 60 andar. Ainda foram observados outros sinais clínicos como hematúria em $3 / 43$ casos sendo $2 / 3$ decorrente de queda acima do 60 andar; cistite traumática em $1 / 43$ casos e alterações neurológicas em 4/43, todos decorrentes de quedas até o 60 andar.

Dos 43 gatos que foram atendidos, 22 (51,16\%) receberam tratamento emergencial, 19 (44,19\%) não emergencial e 6 vieram a óbito, sendo que 2/6 (4,65\%) já chegaram mortos.

Foi observada alteração na rotina da casa em $17 / 43$ (39,53\%) dos casos e em 26/43 (60,46\%) dos casos não houve nenhuma alteração na rotina da casa.

De maneira a resumir os tipos de lesões e fraturas sofridas pelos felinos, segundo as duas classes de altura, são apresentados os Quadros 1 e 2 .

Quadro 1. Lesões ortopédicas e sua localização observadas em 43 gatos que sofreram queda de altura equivalente ao $6^{\circ}$ andar e de altura igual ou superior ao 70 andar, no período de janeiro de 2010 a dezembro de 2012, no Município do Rio de Janeiro.

\begin{tabular}{|c|c|c|c|c|c|c|c|c|c|c|c|}
\hline $\begin{array}{c}\text { Andar } \\
\text { Lesão }\end{array}$ & $\begin{array}{c}\text { Fratura } \\
\text { Membro } \\
\text { torácico }\end{array}$ & $\begin{array}{c}\text { Fratura } \\
\text { Membro } \\
\text { pélvico }\end{array}$ & $\begin{array}{c}\text { Luxação } \\
\text { MT }\end{array}$ & $\begin{array}{c}\text { Luxação } \\
\text { MP }\end{array}$ & $\begin{array}{c}\text { Fratura } \\
\text { pelve }\end{array}$ & $\begin{array}{c}\text { Fratura } \\
\text { coluna }\end{array}$ & $\begin{array}{c}\text { Luxação } \\
\text { coluna }\end{array}$ & $\begin{array}{c}\text { Luxação } \\
\text { articulação } \\
\text { têmporo- } \\
\text { mandibular }\end{array}$ & $\begin{array}{c}\text { Fratura } \\
\text { sínfise }\end{array}$ & $\begin{array}{c}\text { Fratura } \\
\text { mandíbula }\end{array}$ & $\begin{array}{c}\text { Fratura } \\
\text { canino }\end{array}$ \\
\hline $\begin{array}{c}\text { Até 60 } \\
\text { andar }\end{array}$ & $12 / 35$ & $9 / 35$ & $2 / 35$ & $2 / 35$ & --- & --- & $4 / 35$ & $3 / 35$ & $2 / 35$ & $3 / 35$ & $2 / 35$ \\
\hline $\begin{array}{c}70 \text { andar } \\
\text { ou mais }\end{array}$ & $6 / 8$ & $4 / 8$ & --- & --- & $2 / 8$ & $1 / 8$ & --- & $1 / 8$ & $1 / 8$ & -- & -- \\
\hline
\end{tabular}

Quadro 2. Lesões traumáticas e sintomas observados em 43 gatos que sofreram queda de altura equivalente ao 60 andar e igual ou superior ao 70 andar, no período de janeiro de 2010 a maio de 2012, no Município do Rio de Janeiro.

\begin{tabular}{|c|c|c|}
\hline Lesões e sintomas & Até 60 andar & 70 andar ou mais \\
\hline Pneumotórax & $3 / 35$ & $4 / 8$ \\
\hline Contusão pulmonar & $7 / 35$ & $4 / 8$ \\
\hline Fístula oronasal & $7 / 35$ & $1 / 8$ \\
\hline Lesão na língua & $4 / 35$ & $1 / 8$ \\
\hline Escoriações na face & $18 / 35$ & $1 / 8$ \\
\hline Epistaxe & $8 / 35$ & $3 / 8$ \\
\hline Insuficiência renal aguda & $1 / 35$ & $1 / 8$ \\
\hline Alteração neurológica & $4 / 35$ & $0 / 8$ \\
\hline Pancreatite & $1 / 35$ & $0 / 8$ \\
\hline Hematúria & $1 / 35$ & $2 / 8$ \\
\hline Dispnéia & $5 / 35$ & $3 / 8$ \\
\hline
\end{tabular}




\section{Discussão}

A média de idade dos felinos que sofreram queda ( 2 anos e 9 meses) e que fazem parte da presente pesquisa é aquela referida nos trabalhos de Whitney \& Mehlhaff (1987); Pratschke \& Kirby (2002); Boundrieau (2004); Vnuk et al. (2004) e Crystal (2009) os quais informam que a maioria dos felinos envolvidos em queda de grande altura são relativamente jovens (menos de 3 anos de idade) e inexperientes em relação ao ambiente

Os dados obtidos com $60,47 \%$ de queda de felinos machos concordam com os determinados por Barbosa \& Schossler (2009) ao fazerem um estudo retrospectivo de 1997 a 2006 sobre a luxação coxofemoral traumática em cães e gatos, atendidos no Hospital Veterinário Universitário da Universidade Federal de Santa Maria, onde os autores afirmaram que a queda é maior em animais adultos e em machos.

Gordon et al. (1993) diz que 72\% dos cães que sofreram queda eram inteiros, sendo este um fator relevante na ocorrência das mesmas. Este dado contrapõe ao observado neste estudo, onde a castração não aumentou ou diminuiu de forma significativa o percentual de queda entre os felinos. As quedas em cães inteiros está relacionada com períodos de cio, entretanto este dado é de difícil obtenção em relação aos gatos, pois a maioria dos proprietários tem dificuldade em identificar se o gato está no cio.

Entretanto, embora seja um dado de difícil avaliação, a queda dos gatos machos inteiros pode estar relacionada ao fato destes animais perceberem a presença de fêmeas no cio, e então, tentarem sair para acasalar, ficando assim, mais expostos ao risco de queda.

Neste estudo, as quedas ocorreram em números semelhantes durante o período do dia e da noite. Este dado pode ser explicado pelo fato de que a clínica veterinária onde o estudo foi realizado, é referência no atendimento de felinos e funciona 24 horas, portanto, recebe emergências a qualquer horário. Os dados obtidos na presente pesquisa são diferentes dos obtidos por Whitney \& Mehlhaff (1987) e Gordon et al. (1993), os quais relatam que a maior incidência das quedas se dá no período noturno, relacionando este fato a maior atividade dos gatos nesse período.

Embora não significativo, pode-se afirmar que a maioria das quedas foram observadas nos fins de semana, quando usualmente as pessoas se encontram mais em seus lares, deixando as janelas abertas, concordando com os resultados obtidos por Whitney \& Mehlhaff (1987); Gordon et al. (1993) e Faria (2003).

Neste estudo não foi notada influência sazonal como relatada nos estudos de Smith et al. (1975); Robinson (1976); Warner \& Demling (1986) e Gordon et al. (1993). Os resultados podem estar relacionados ao fato de que as estações do ano não ocorrerem de forma tão definida no Município do Rio de Janeiro, e como as temperaturas não variam tanto, as janelas são deixadas abertas durante todo o ano, o que não ocorre em países onde o inverno é rigoroso.

Verificamos a ocorrência de quedas, mesmo em residências que tinham todas as janelas teladas. Este dado pode ser explicado pelo fato de que muitas vezes as telas instaladas não são adequadas, possuindo malha de tamanho que permite que um gato menor passe por ela. Muitas vezes também, as telas não são adequadamente instaladas, havendo frestas ou malhas frouxas permitindo a passagem dos gatos. Frequentemente, também se observa que as telas não sofrem manutenção permanente, e com o tempo acabam sofrendo desgaste e são facilmente danificadas. Este dado não foi relatado anteriormente.

Os tipos de lesões e fraturas observadas estão de acordo com as descritas por outros autores (Whitney \& Mehlhaff, 1987; Gordon et al., 1993; Faria, 2003; Vnuk et al., 2004; Crystal, 2009).

Lesões em órgãos do sistema esplâncnico não foram relatadas anteriormente. Neste estudo, foram observadas em decorrência de quedas acima do $6^{\circ}$ andar.

Devido ao fato de muitos gatos ficarem assustados e estressados com mudanças ealterações na sua rotina, estas podem estar relacionadas diretamente com a ocorrência de quedas. Este dado não foi avaliado em nenhum estudo prévio.

Neste estudo foi observado que muitas quedas ocorreram em função de alterações na rotina da casa como por exemplo, festas, reuniões, obras, mudança, chegada a nova residência, confinamento do gato em local restrito, presença de visita, consertos e dedetização. Essas alterações geraram estresse para o gato, fazendo com que tentasse fugir da situação estressante, ocorrendo então a queda. 


\section{Conclusões}

A idade é um fator importante na ocorrência de queda de grande altura e os animais mais jovens são os mais sujeitos ao acidente.

A alteração na rotina da casa deve ser considerada, pois pode gerar estresse, e para tentar fugir da situação estressante, o gato se expõe ao risco de queda.

A ausência de telas de proteção é um fator que favorece a queda dos animais. Redes de proteção adequadas devem ser instaladas em todas as janelas da residência.

O estado reprodutivo pode influenciar a ocorrência da queda, pois os animais inteiros podem tentar sair em épocas de cio, para acasalar, ficando mais expostos ao risco de queda.

As fraturas e lesões de face ocorrem com grande frequência. Devemos sempre estar atentos para identificar lesões pulmonares, pois estas quando não adequadamente tratadas, podem levar os gatos a óbito, como também, as lesões em órgãos do sistema esplâncnico.

Embora os gatos sejam animais de hábito noturno, as quedas ocorrem em qualquer período do dia, bem como em qualquer época do ano indistintamente, não sendo verificada uma sazonalidade nos casos atendidos.

As quedas tiveram uma ocorrência maior nos fins-de-semana. Este fato pode estar relacionado ao fato das pessoas ficarem mais em casa nos referidos dias.

\section{Referências}

Barbosa, A. L. T., \& Schossler, J. E. W. (2009). Luxação coxofemoral traumática em cães e gatos: estudo retrospectivo (1997-2006). Ciência Rural, 39(6), 1823-1829. http://dx.doi.org/10.1590/S0103-84782009000600028.

Boundrieau, R. J. (2004, September 10-12). High-rise syndrome in cats. In A. Vezzoni \& M. Schramm(Eds.), Congress of The European Society of Veterinary Orthopaedics and Traumatology: Vol. 12. (pp. 24-25). Munich: ESVOT.

Crystal, M. A. (2009). Síndrome da queda de grande altura. In G. D. Norsworthy, M. A. Crystal, S. F. Grace, \& L. P. Tilley (Eds.), O paciente felino (3a ed. pp. 134-135). São Paulo: Manole.

Faria, M. L. E. (2003) Síndrome do gato para-quedista: traumatismo por queda. In H. J. M. Souza (Ed.), Coletâneas em medicina e cirurgia felina (pp. 405-422). Rio de Janeiro: L.F. Livros de Veterinária.

Gordon, L. E., Thacher, C., \& Kapatkin, A. (1993). High-rise syndrome in dogs: 81 cases (1985-1991). Journal of the American Veterinary Medical Association, 202(1), 118-122. PMid:8420897.

Pratschke, K. M., \& Kirby, B. M. (2002). High rise syndrome with impalement in three cats. The Journal of Small Animal Practice, 43(6), 261-264. http://dx.doi.org/10.1111/j.1748-5827.2002.tb00070.x. PMid:12074291.

Robinson, G. W. (1976). The right rise trauma syndrome in cats. Feline Practice, 6(1), 40-43.

Smith, M. D., Burrington, J. D., \& Woolf, A. D. (1975). Injuries in children sustained in free-falls: an analysis of 66 cases. The Journal of Trauma, 15(11), 987-991. PMid:1195446.

Vnuk, D., Pirkic, B., Maticic, D., Radisic, B., Stejskal, M., Babic, T., Kreszinger, M., \& Lemo, N. (2004). Feline highrise syndrome: 119 cases (1998-2001). Journal of Feline Medicine and Surgery, 6(5), 305-312. http://dx.doi. org/10.1016/j.jfms.2003.07.001. PMid:15363762.

Warner, K. G., \& Demling, R. H. (1986). The pathophysiology of free-fall injury. Annals of Emergency Medicine, 15(9), 1088-1093. http://dx.doi.org/10.1016/S0196-0644(86)80134-2. PMid:3740599.

Whitney, W. O., \& Mehlhaff, C. J. (1987). High-rise syndrome in cats. Journal of the American Veterinary Medical Association, 191(11), 1399-1403. PMid:3692980. 